\title{
The study of atmospheric vortex streets over the North-West Pacific from meteorological satellite imagery
}

\author{
M. G. Aleksanina, A. C. Eremenko, S. E. Dyakov, A. V. Kazansky, V. A. Levin \\ Institute of Automation and Control Processes, FEB RAS, Vladivostok, 690041, \\ Russia \\ E-mail: margeo@mail.ru
}

\begin{abstract}
The results of satellite monitoring of atmospheric vortex streets behind Jeju Island in the Korean Strait in the winter of 2015 and behind Kuril Islands in the spring of 2012 are presented. The main hydrodynamic parameters of atmospheric vortex streets were calculated using satellite data. These are the numbers of Reynolds, Strouhal, Froude as well as the speed of vortex drift and vortex generation time by the calculation of geometric parameters. The characteristic obstacle typical size was chosen at the height of the inversion layer upper boundary based on the vertical atmosphere temperature profile. We compared the results with a widely used approach (the "dividing-streamline" concept) that is based on an estimate of the height of the airflow line separating the horizontal flow around the obstacle from the flow through it. Shown that the vortex streets appear to arise in the inversion layer, and the characteristic obstacle size must correspond to the size of the section at the height that is below the inversion layer upper boundary, but above the island base.
\end{abstract}

\section{Introduction}

With the advent of meteorological satellites, it became possible to regularly observe atmospheric vortex streets in different parts of the world. A large number of papers are devoted to the atmospheric vortex streets in the northeast Atlantic [1-3]. In north-western part of the Pacific Ocean the atmospheric vortex streets are observed behind Jeju Island every winter period [4]. It should be noted that most of the atmospheric vortex street studies rests on the model calculations and the laboratory study results [5-8]. Based on this numerical simulation, importance of density stratification to observe atmospheric vortices streets were shown [9]. Despite the numerous studies, there is no single point of view yet for the mechanism of formation in the real atmospheric vortex streets. The phenomenon can be observed only for limited values of Reynolds number. The street sizes depend on the size of the body in stream, and there is a linear relationship between the street width and the distance between neighbouring vortices [10].

This work is dedicated to results of satellite observations of the atmospheric vortex street behind Jeju Island in the Korean Strait in the winter of 2015 and behind Kuril Islands in the spring of 2012. The available satellite observations made it possible to calculate the main geometric and hydrodynamic parameters responsible for the formation of atmospheric vortex streets (AVS). It was possible to trace the initial stage of the formation in the real atmospheric vortex streets and to calculate the vortex generation frequency of using the vortex speed, the street width and step. It is assumed that the vortex streets appear in the stratified atmosphere in the cases under consideration, with the Reynolds numbers being of the order of $10^{10}$. 


\section{Data and methods used}

Jeju Island is an island of volcanic origin, Hallasan volcano is situated here, the highest mountain in South Korea $(1,950 \mathrm{~m})$. The island length is about $75 \mathrm{~km}$. The atmospheric vortex streets are observed here annually, from January to February. In January 2015, the atmospheric vortex streets were observed in satellite images from 7 to 31 January. The most stable and smooth cloud vortex streets were observed on January 8, 2015. The minimum time interval between satellite observations was 30 minutes.

Kuril Islands are a chain of volcanic islands between the peninsula of Kamchatka and Hokkaido Island. Up to 10 atmospheric vortex streets of different scale were observed in satellite images on April 16, 2012 (Figure 1). With the available resolution $(1.1 \mathrm{~km}$, the AVHRR/NOAA radiometer, and the $250 \mathrm{~m}$, MODIS/Aqua radiometer), the atmospheric vortex street were observed most clearly behind the four islands of the middle Kurile string: Matua Island, Rasshua Island, Ketoy Island, and Simushir Island. The minimum time interval between satellite observations was 13 minutes.

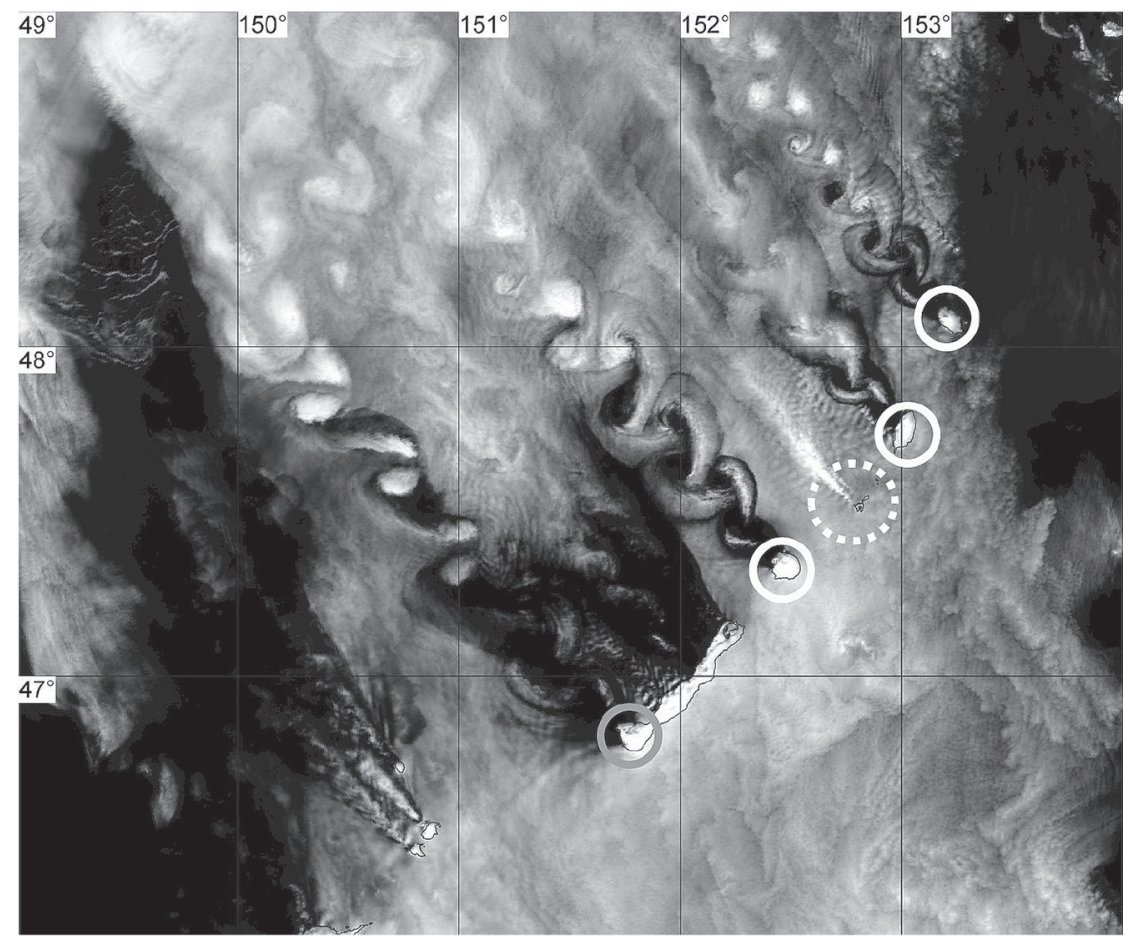

Figure 1. The image in a visible spectral channel on April 16, 2012 from the MODIS/Aqua satellite (02:16:59 UTC)

To calculate the vertical atmosphere temperature and humidity profiles, the data of the ATOVS radiometers (the NOAA-15, $-18,-19$ satellites) were adapted for parallel processing with the European Space Agency software packages: AAPP, RTTOV and MetOffice-1Dvar. These packages are used in European weather forecasts. The profiles are calculated at 43 standard levels vertically. For each point, the most suitable standard initial approximation of the profile from the values available in the RTTOV package was determined depending on the geographical coordinates and season. We selected only the profiles that had passed the strict accuracy check.

To calculate the ongoing air flow speed, the advanced method of cross correlation of the formation of the cloud marker drift in the satellite image sequence was used [11]. It was assumed that the flow speed was constant vertically from the surface to the inversion upper boundary. The parameters of the atmospheric vortex streets and the drift of the discrete vortices were calculated visually.

\section{The theory and experiments}

In a real atmosphere, cloud vortex streets are observed most frequently behind volcanic islands in the sea and are generated when the lower atmosphere layer is bounded from above by the expressed inversion located much lower than the top of the island or ridge [5]. The inversion prevents to flow above 
the obstacle, and the sidewall flow-around is implemented as a result. It is assumed that there is an interface between two types of flow around an obstacle, the level of which is called the "dividing streamline" and which lies above the inversion level. If strong stratification is presented in the atmosphere, the inversion is expressed sufficiently and mountain is sufficiently high too, then you should expect the vortex shedding. Under these conditions, the flow below the dividing streamline is forced to go around the island in almost horizontal planes with the possible generation of the vortices with the vertical axis. The indicators of these regimes are the Reynolds and Strouhal dimensionless numbers. To calculate them, the estimates of the real wind speed, direction and of the effective obstacle (the island) diameter $d$ are required.

The Strouhal number $(\mathrm{St})$ related with Reynolds number $(\mathrm{Re})$ for the flow generating the street is a dimensionless frequency of the vortex generation [10]:

$$
\operatorname{Re}=\frac{\rho V_{w} d}{\mu},
$$

where $\rho$ is the medium density, $\mu$ is the medium dynamic viscosity coefficient, $V_{w}$ is the speed of the flow oncoming at the obstacle, and $d$ is the obstacle linear size. The Strouhal number value St for an obstacle in the form of a circular cylinder in the so-called supercritical region ( $\operatorname{Re} \gg 10^{7}$ ) differs in papers of different researchers and corresponds to the range of $0.22-0.30[12,13]$. Strictly speaking, laboratory experiments that would correspond to our cases when $\operatorname{Re} \sim 10^{10}$ were absent, since the states when $\operatorname{Re} \gg 10^{7}$ are not generated, so we will have to rely on these estimates. The Strouhal number is related to the vortex shedding frequency $f$ with the relation [3]

$$
\mathrm{St}=\frac{f d}{V_{w}}
$$

Where $V_{w}$ is the oncoming flow speed; $f$ is the vortex generation frequency; $d$ is the typical obstacle linear size. After calculating the Strouhal number, we can calculate the vortex generation frequency $f$

$$
f \approx \frac{\mathrm{St} \cdot V_{w}}{d}
$$

On the other hand, the vortex generation frequency $f$ is determined through the vortex displacement speed $V_{v}[3]$ :

$$
f \approx \frac{V_{v}}{a},
$$

where $a$ is the distance between the vortices of the same rotation sign. Having the satellite image sequence with the time interval, for which the cloudiness keeps its figure, you can calculate the rates of the vortex displacement speeds $V_{v}$, the oncoming flow speed $V_{w}$ and the rest geometric characteristics of the vortex streets: the transverse size $b$ and the distance between two vortices of the same sign $a$ (Figure 2).

In this case, it is possible to obtain the relationships allowing calculating Strouhal number, St, directly:

$$
\mathrm{St} \approx \frac{V_{v} d}{a V_{w}} \cong \frac{V_{v} / V_{w}}{d / a} .
$$

To calculate the dimensionless Reynolds number $\mathrm{Re}$, we take the relative air density as $\rho=1.019 \mathrm{~kg} / \mathrm{m}^{3}$, and the air viscosity $\mu$ as $1.68 \cdot 10^{-5} \mathrm{~Pa} \cdot \mathrm{s}$. These quantities may be considered as constants in space and time. The vertical temperature profiles above the studied islands, Jeju Island for January 8, 2015 and for Kuril Islands - Matua, Rasshua, Ketori and Simushir Islands - for April 16, 2012, showed the inversion presence at the height much lower than the height of volcanic islands (Figure 3 and 4).

The typical obstacle size $d$ was the width of the island profile that is transverse to the flow at the height corresponding to the inversion upper boundary. To do this, profiles of the islands perpendicular to the flow were constructed, according to the satellite topography STRM (https://www2.jpl.nasa. 
gov/srtm/) with a spatial resolution of $90 \mathrm{~m}$ (Figure 5). An estimate of the inversion upper boundary height was obtained as follows. In the inversion layer, the temperature was almost constant, but an almost linear decrease was observed above this layer. The intersection of the temperature linear approximation above the inversion layer with the vertical line of constant temperature in the inversion layer gave the heights for both Kuril and Jeju Islands. The heights in the Kuril Islands were almost identical and slightly fluctuated around the level of $520 \mathrm{~m}$. For each island, the profiles in the oncoming flow in front of the island and immediately after was almost identical and differed only by a slight shift in the temperature scale: about $0.3{ }^{\circ} \mathrm{C}$ (the temperature was higher behind the island).

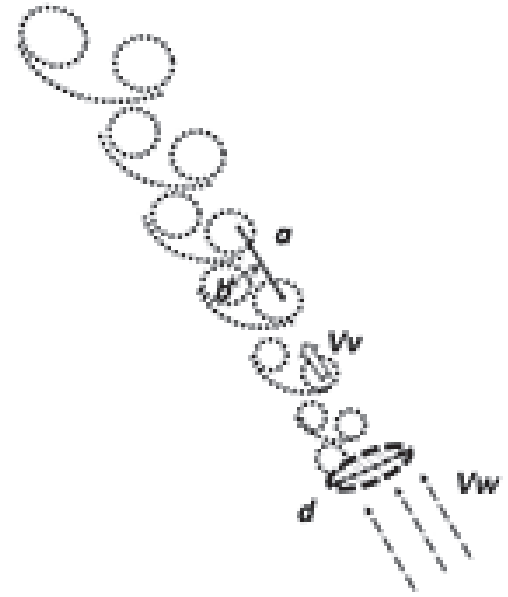

Figure 2. Diagram of the atmospheric vortex street

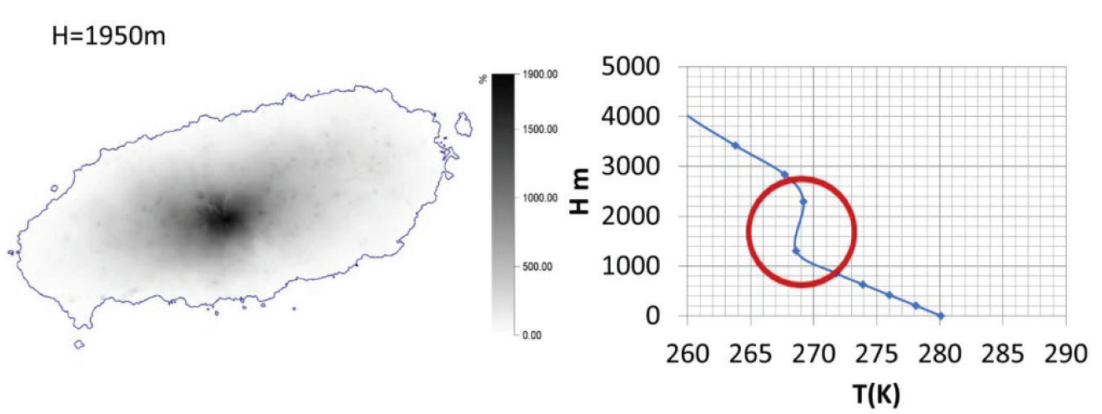

Figure 3. Atmospheric temperature profile in front of Jeju Island for January 8, 2015 and the island height (a)

$H=1446 m$

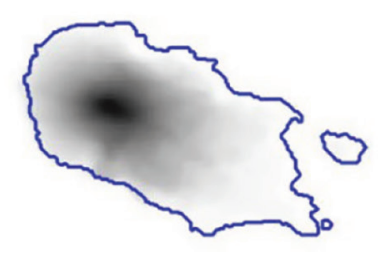

(b)

$H=948 \mathrm{~m}$

(c) $\mathrm{H}=1166 \mathrm{~m}$
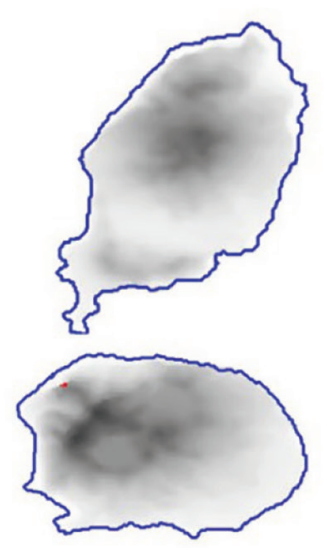

(d)
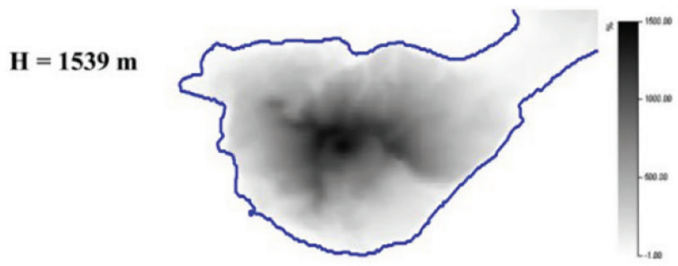
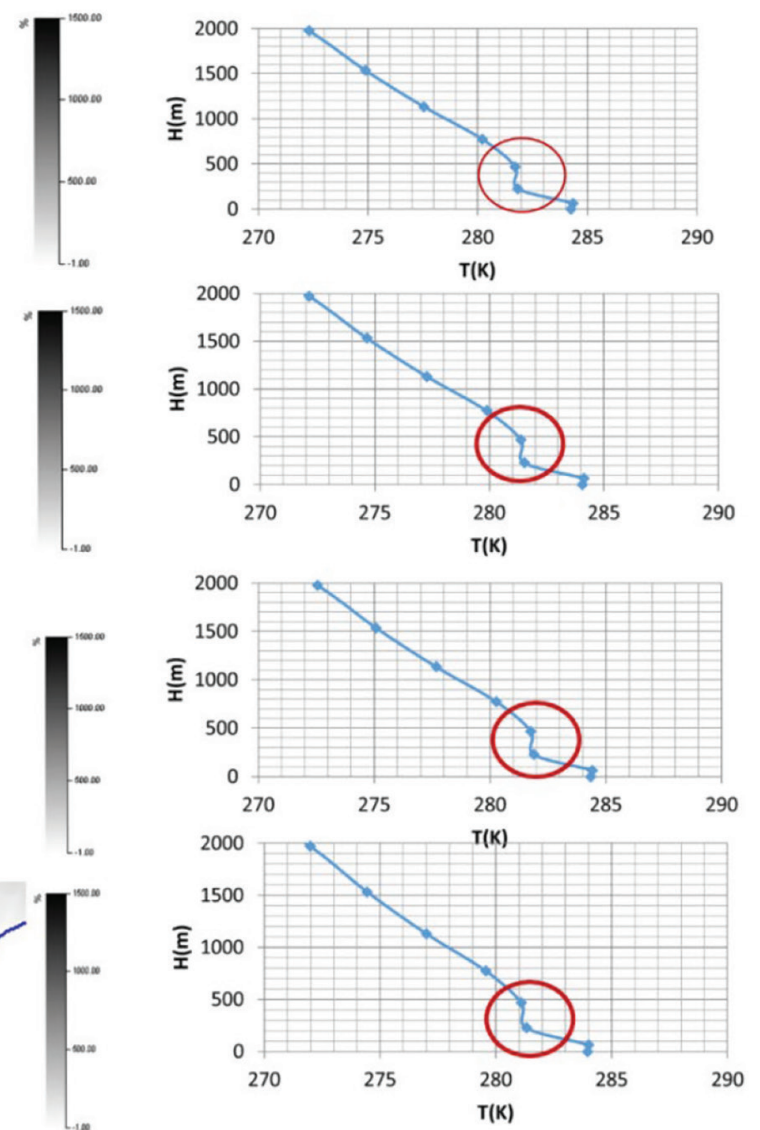

Figure 4. Atmospheric temperature profiles in front of the Kurile Islands, for April 16, 2012 and the height of the islands: (a) Matua; (b) Rasshua; (c) Ketoy; (d) Simushir. 


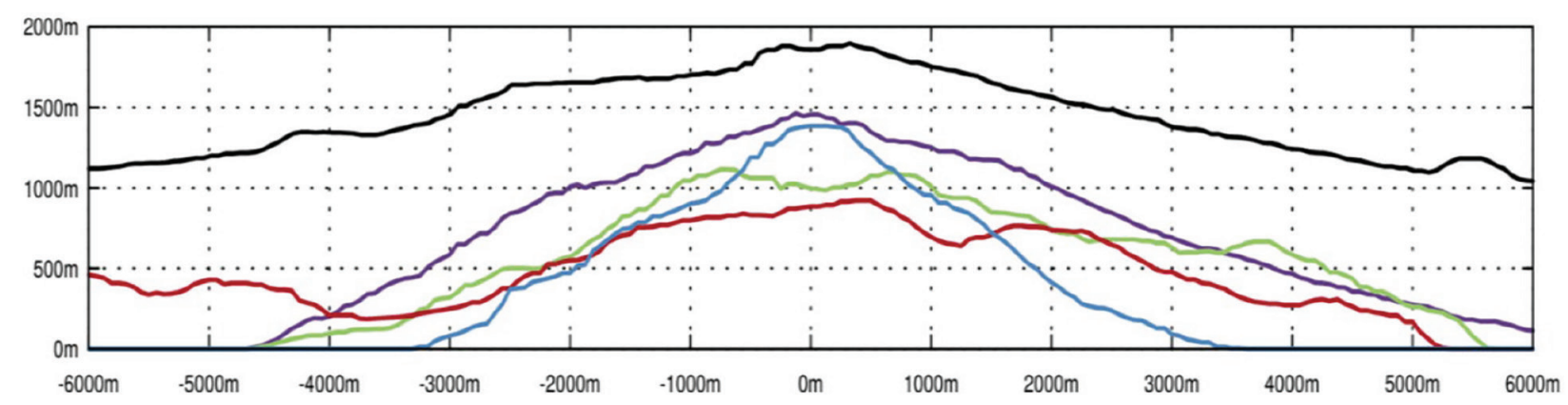

Figure 5. Profiles of the islands in the plane perpendicular to the wind: black colour - Jeju, lilac colour - Simushir, green colour - Keto, red colour - Rasshua, and blue colour - Matua.

The inversion heights were compared with the height of the airflow line separating the horizontal flow around the obstacle from the flow through it, $h_{c}$. The formulas given in the paper [5] were used for these calculations. The potential temperature $\theta$ was calculated using the vertical temperature profiles. Then the Brunt-Väisälä frequency $N$ was calculated for each level of height $z$ represented in the vertical profiles:

$$
N=\left(\frac{g \delta \theta}{\theta_{0} \delta z}\right)^{1 / 2},
$$

where $g$ is the acceleration of gravity, $\theta_{0}$ is the average potential temperature in the layer for which the balance was been calculated (7). Having the calculated speed of the oncoming flow $V_{w}$, the obstacle height (the island height), the Brunt-Väisälä frequency, the integral according to the expression [14], was calculated to obtain the characteristic flow height $h_{c}$ :

$$
\frac{1}{2} V_{w}^{2}\left(h_{c}\right)=\int_{h_{c}}^{H} N^{2}(z) \cdot(H-z) \mathrm{d} z .
$$

Since the height $h_{c}$ is unknown, it was computed by picking the $h_{c}$ value until left and right parts of the integral were the same. After obtaining the height $h_{c}$, the Froude number was calculated for each island:

$$
F r=1-\frac{h_{c}}{H}
$$

Using atmosphere profiles and the oncoming flow speed, the Froude numbers and heights were calculated where the flow was split (table 1). The atmosphere profiles for the Kuril Islands are identical in space and have in fact the same height of the inversion upper level. It is reasonable to expect that the flow dividing streamline will also have the same height. However, the $h_{c}$ scatter is considerable (from 300 to $700 \mathrm{~m}$ ). At the same time, the average $h_{c}$ value is close to the value of the inversion upper boundary. Apparently, these values are identical, but the estimate that uses the atmosphere temperature profiles is more reliable.

Table 1. The calculated characteristic heights $h_{c}$, the Froude numbers $F r$ and the characteristic linear obstacle size $d$ in the dividing streamline.

\begin{tabular}{lccccccc}
\hline Island & $h_{c}, \mathrm{~m}$ & $F r$ & $d, \mathrm{~m}$ & $a \times 10^{3}, \mathrm{~m}$ & $V_{v}, \mathrm{~m} / \mathrm{s}$ & $f \times 10^{-3}$ & $T$, vortex per hour \\
\hline Jeju & 925 & 0.52 & 14800 & $65-110$ & 12.5 & $0.19-0.11$ & $1.44-2.44$ \\
Matua & 578 & 0.6 & 3570 & $19-22$ & 8.5 & $0.70-0.38$ & $0.39-0.72$ \\
Rasshua & 305 & 0.68 & 10252 & $17-27$ & 11.15 & $0.65-0.41$ & $0.42-0.67$ \\
Ketoy & 512 & 0.56 & 5486 & $25-30$ & 8.75 & $0.35-0.29$ & $0.79-0.95$ \\
Simushir & 712 & 0.54 & 5676 & $33.7-36.5$ & 8.033 & $0.28-0.22$ & $0.96-1.26$ \\
\hline
\end{tabular}

The vortex frequency $f$ is determined according to the formula (4) through the vortex displacement speed $V_{v}$ and the distance between two vortices of the same rotation sign $-a$ [3]. The calculations 
of the vortex shedding frequency and of the vortex period $T$ based on the satellite data for the vortex streets are provided in table 1 .

Table 2. The Strouhal number St and Reynolds number Re, the characteristic obstacle size $d$ for calculation based on the temperature inversion height $h_{i n v}$ and on the dividing streamline height $h_{c}$

\begin{tabular}{lcccccccccc}
\hline Island & $V_{w}, \mathrm{~m} / \mathrm{s}$ & $V_{v}, \mathrm{~m} / \mathrm{s}$ & $h_{i n v}, \mathrm{~m}$ & $d_{i n v} \times 10^{3}, \mathrm{~m}$ & $\mathrm{St}_{i n v}$ & $\mathrm{Re}_{i n v} \times 10^{10}$ & $h_{c}, \mathrm{~m}$ & $d_{h c} \times 10^{3}, \mathrm{~m}$ & $\mathrm{St}_{h c}$ & $\mathrm{Re}_{h c} \times 10^{10}$ \\
\hline Jeju & 17 & 12.5 & 1050 & 12.49 & 0.14 & 1.28 & 925 & 14.80 & 0.16 & 1.52 \\
Matua & 14 & 8.5 & 525 & 3.68 & 0.18 & 0.31 & 578 & 3.57 & 0.18 & 0.30 \\
Rasshua & 14 & 11.5 & 525 & 5.00 & 0.24 & 0.42 & 305 & 10.25 & 0.51 & 0.87 \\
Ketoy & 14 & 8.7 & 525 & 5.42 & 0.26 & 0.46 & 512 & 5.49 & 0.26 & 0.46 \\
Simushir & 14 & 8.0 & 525 & 6.80 & 0.20 & 0.57 & 712 & 5.68 & 0.17 & 0.48 \\
\hline
\end{tabular}

According to the relationship (5), we can directly calculate the dimensionless quantities characterizing the stability of the vortex streets, the Reynolds and Strouhal numbers. Table 2 provides the values of the Reynolds and Strouhal numbers calculated for the characteristic obstacle diameter $d$. In one case, the diameter $d_{i n v}$ was taken at the height $h_{i n v}$ of the atmosphere profile temperature inversion and in the other case $d_{h c}$ was taken at the height $h_{c}$ of the forking of the flow around the obstacle.

\section{Results and discussion}

For classical vortex streets formed for the flow around a circular cylinder, six modes are known limited by the Reynolds number up to $10^{7}$ [15]. For Reynolds numbers above $10^{7}$, a supercritical turbulence region arises is also characterized by presence of the vortex street, but the geometric relationships of the classical Kármán streets may not be fulfilled for this street.

You can see from table 2 that the Strouhal number St is in most cases smaller than the values corresponding to the supercritical Reynolds numbers, i.e. the number $S t$ takes on a value in the range $0.22-0.3$ for $\operatorname{Re}>10^{7}$. Probably, this is because the estimate of the characteristic obstacle size $d$ is not quite correct. The vortices are generated in the layer from the surface to the inversion upper boundary, therefore, it is necessary to use some characteristic size located below the inversion layer as an effective obstacle diameter $d$.

This relationship of the vortex street geometric parameters does not correspond to the classical relationships of the Kármán streets for the Reynolds numbers up to $200(\operatorname{Re}<200)$. There must be other relations in the supercritical turbulence region at Reynolds numbers greater than $10^{7}$. In addition, the values of the Strouhal number as non-dimensional indicator of the vortex shedding frequency depend on the characteristic obstacle diameter $d$, which should be taken below the temperature inversion line of the atmosphere profile.

Kuril Islands case is interesting due to the observed an atmospheric vortex street generated over an obstacle chain in contrast to isolated ones. However, Kuril Islands contain the islands (the Ushishir Islands), behind which there was a "disturbance 0 in the form of a cloud strip, which is not the vortex street (Figure 1). The maximum height of this island is $401 \mathrm{~m}$ that is below the inversion level of $525 \mathrm{~m}$. It is also worth to mention that the estimation of the heights using the upper part of the atmospheric vortex streets shows values in the range from 2 to $2.5 \mathrm{~km}$ that are significantly higher than the inversion level of $525 \mathrm{~m}$ obtained from the temperature and humidity profiles. This case supports our idea to choose the obstacle typical size at the height of inversion layer. Following such choice the obstacle turns out to be the chain of isolated islands separated by wide gaps able to produce atmospheric vortex streets. Otherwise, using their bases with narrow straits, we would observe turbulent wakes behind a grid like that shown in [16]).

\section{Conclusions}

Using only the satellite monitoring data for the atmospheric vortex streets behind the islands of the Far Eastern region, the main hydrodynamic parameters were calculated: the vortex frequencies, the Strouhal and Reynolds numbers. For their calculation, the characteristic obstacle sizes were estimated by two methods. The first method is based on calculating the "dividing streamline" height $h_{c}$ [5], and on estimating the horizontal size of the island vertical section at this height $d_{h c}$. The second method 
is based on calculating the height $h_{i n v}$ of the inversion layer upper edge using the satellite atmospheric vertical temperature profiles with an estimate of the similar characteristic island size.

The atmosphere profiles for the Kuril Islands are identical in space and have in fact the same height of the inversion upper level. This height almost coincided with the average height $h_{c}$, indicating the possible identity of these two ways. However, because of the wide variation in the values of $h_{c}$ in Kuril Islands, the first method is less reliable. The islands, which height was below the inversion layer of the $h_{i n v}$, did not generate the streets.

The vortex streets appear to arise in the inversion layer, and the characteristic obstacle size must correspond to the size of the section at the height that is below the inversion layer upper boundary, but above the island base. This is indirectly indicated by the Strouhal numbers that are smaller than the corresponding estimates for the supercritical Reynolds numbers, although there are no laboratory estimates of the Strouhal numbers for the obtained Reynolds numbers $\left(\sim 10^{10}\right)$.

\section{Acknowledgements}

This work was supported by Presidium RAS, Program "Fundamental aspects of supercomputing for simulating complex practical problems".

\section{References}

[1] Couvelard X., Caldeira R. M.A., Araújo I.B., Tomé R., Wind mediated vorticity-generation and eddyconfinement, leeward of the Madeira Island: 2008 numerical case study, Dynamics of Atmospheres and Oceans, 2012, Vol. 58, pp. 128-149.

[2] Caldeira R. M.A., Tomé R., Wake response to an ocean-feedback mechanism: Madeira Island case study, Boundary-Layer Meteorology, 2013, Vol. 148, pp. 419-436.

[3] Nunalee C. G., Basu S., On the periodicity of atmospheric von Kármán vortex streets, Environmental Fluid Mechanics, 2014, Vol. 14, pp. 1335-1355.

[4] Chung Y.S., Kim H. S., Mountain-generated vortex streets over the Korea South Sea, Intern. J. Remote Sensing, 2007, Vol. 29, Issue 3, pp. 867-877.

[5] Etling D., On atmospheric vortex streets in the wake of large islands, Meteorology and Atmospheric Physics, 1989, Vol. 41, pp. 157-164.

[6] Young G.S., Zawislak J., An observational study of vortex spacing in island wake vortex streets, Monthly Weather Review, 2006, Vol. 134, pp. 2285-2294.

[7] Heinze R., Raasch S., Etling D., The structure of Karman vortex streets in the atmospheric boundary layer derived from large eddy simulation, Meteorologische Zeitschrift, 2012, Vol. 21, pp. 221-237.

[8] Ito J., Niino H., Atmospheric Kármán Vortex Shedding from Jeju Island, East China Sea: A Numerical Study, Monthly Weather Review, 2016, Vol. 144(1), pp. 139-148.

[9] Epifanio C., Rotunno R., The Dynamics of Orographic Wake Formation in Flows with Upstream Blocking, Atmospheric Sciences, 2005, Vol. 62(9), pp. 3127-3150.

[10] Batchelor G. K., An Introduction to Fluid Dynamics, Cambridge: Cambridge University Press, 2000, 615 p.

[11] Aleksanin A. I., Aleksanina M. G., Karnatskii A. Yu., Automatic computation of sea surface velocities on a sequence of satellite images, Sovremennye problemy distantsionnogo zondirovaniya Zemli iz kosmosa, 2013, Vol. 10(2), pp. 131-142.

[12] Blevins R. D., Flow-induced Vibration, New York: Van Nostrand Reinhold Co., 1990, 451 p.

[13] Achenbach E., Heinecke E., On vortex shedding from smooth and rough cylinders in the range of Reynolds numbers $6 \cdot 10^{3}$ to $5 \cdot 10^{6}$, J. Fluid Mechanics, 1981, Vol. 109, pp. 239-251.

[14] Snyder W. H., Thompson R. S., Eskridge R.E., Lawson R.E., Castro L.P., Lee J.T.， Hunt J.C.R., Ogawa Y., The structure of strongly stratified flow over hills: dividing streamline concept, J. Fluid Mechanics, 1985, Vol. 152, pp. 249-288.

[15] Lienhard J.H., Synopsis of Lift, Drag and Vortex Frequency Data for Rigid Circular Cylinders. Pullman, Washington: Technical Extension Service, Washington State University, 1966, Ser. Washington State University, College of Engineering, Research Division Bulletin 300, 32 p.

[16] Wu J.Z., Ma H.Y., Zhou M.D., Vorticity and Vortex Dynamics, Berlin; Heidelberg; New York: Springer, 2006, $13 \mathrm{p}$. 